\title{
PIGMENTOS FOTOSINTÉTICOS DE Stevia rebaudiana Bert. EN CONDICIONES DIFERENCIALES DE LUZ SOLAR Y FERTILIZACIÓN NITROGENADA EN INVERNADERO
}

\author{
PHOTOSYNTHETIC PIGMENTS IN Stevia rebaudiana Bert. UNDER \\ DIFFERENTIAL CONDITIONS OF SUNLIGHT AND NITROGEN \\ FERTILIZATION IN GREENHOUSE
}

\begin{abstract}
Alfredo de Jesús Jarma-Orozco • ajarma@correo.unicordoba.edu.co Ingeniero agrónomo PhD, Profesor Titular, Facultad de Ciencias Agrícolas, Programa de Ingeniería Agronómica, Universidad de Córdoba.
\end{abstract}

Carina Cecilia Cordero-Cordero•ccordero@agrosavia.co Ingeniero Agrónomo, MsC. Agrosavia C.I. Motilonia.

José Alejandro Cleves-Leguízamo•jose.cleves@uptc.edu.co Ingeniero agrónomo, PhD, Profesor Titular, Universidad Pedagógica y Tecnológica de Colombia (UPTC), Facultad Seccional Duitama, Escuela de Administración de Empresas Agropecuarias, Duitama, Colombia.

Autor de correspondencia: ajarma@correo.unicordoba.edu.co

Citación: Jarma-Orozco, A., Cordero-Cordero, C, Cleves-Leguizamo, J. (2022). Pigmentos fotosintéticos en Stevia rebaudiana Bert. en condiciones diferenciales de luz solar y fertilización nitrogenada en invernadero. Revista de Investigación Agraria y Ambiental,13(1), 75 - 88. DOI: https://doi.org/10.22490/21456453.4528

\section{RESUMEN}

Contextualización: El área de siembra del cultivo de estevia ha venido creciendo de manera significativa, debido a las nuevas tendencias de consumo de alimentos naturales, bajos en calorías. Los efectos de las altas radiaciones, debidas al cambio climático global, han evidenciado la necesidad de desarrollar nuevos estudios sobre el comportamiento fisiológico de los pigmentos fotosintéticos de las especies agrícolas.

Vacío de conocimiento: El desconocimiento del comportamiento de los cultivos de estevia frente a los cambios actuales y futuros del ambiente (principalmente ante aumentos de los niveles de radiación solar) hace que los agricultores y productores no obtengan los rendimientos esperados.

Propósito del estudio: El objetivo de este estudio fue analizar el efecto de diferentes niveles de radiación solar y de fertilización nitrogenada, en condiciones controladas, en la producción de pigmentos fotosintéticos de Stevia rebaudiana Bert.

Metodología: El ensayo se estableció mediante un diseñoestadístico completamente al azar (DCA), bajo un arreglo factorial $2 \times 6$, y 16 repeticiones por tratamiento; el factor A correspondió a dos niveles de radiación solar incidente (300 y $1.500 \mu \mathrm{mol}$ de fotones. $\mathrm{m}^{-2} \mathrm{~s}^{-1}$ correspondientes al $20 \%$ y $100 \%$ de radiación respectivamente) y el factor $\mathrm{B}$ a seis dosis de nitrógeno $(0,60,120,180,240$ y $300 \mathrm{~kg} \mathrm{ha}^{-1}$ ). Se evaluó el contenido de clorofilas a, b y total en hojas jóvenes y sanas a los ocho meses después del trasplante.

Resultados y conclusiones: Los resultados evidenciaron que los contenidos de clorofilas a, b y total fueron afectados significativamente por la radiación solar, pero no por los niveles de nitrógeno. En ambos ambientes de 
radiación, los contenidos de clorofila a fueron superiores a los de clorofila b. En el ambiente de menor radiación solar se presentaron los máximos contenidos de los pigmentos fotosintéticos. En el ambiente de $1.500 \mu \mathrm{mol}$ de fotones $\mathrm{m}^{-2} \mathrm{~s}^{-1}$, los contenidos de clorofilas a, b y total fueron menores en las plantas que no se fertilizaron.

Palabras clave: clorofila a; clorofila $b$; clorofila total; nitrógeno; radiación solar

\section{ABSTRACT}

Contextualization: The areas planted with stevia have been growing significantly in response to the new trends in consumption of low-calorie and natural foods. The effects of high radiation levels, due to global climate change, have evidenced the need to development new studies about the physiological behavior of the photosynthetic pigments of agricultural species.

Knowledge gap: The lack of knowledge of the behavior of stevia crops in the context of current and future environmental changes (mainly, the increases in solar radiation levels) means that farmers and producers do not obtain the expected yields.

Purpose: The objective of this study was analyze the effect of different levels of solar radiation and nitrogen fertilization, under greenhouse conditions, on the production of photosynthetic pigment in Stevia rebaudiana Bert.

Methodology: The trial was done using as completely randomized design (CRD), employing a $2 \times 6$ factorial arrangement and 16 replicates per treatment; factor $A$ corresponded to two levels of incident solar radiation (300 and $1.500 \mu \mathrm{mol}$ photons. $\mathrm{m}^{-2}$ $\mathrm{s}^{-1}$ corresponding to $20 \%$ and $100 \%$ radiation respectively) and factor $\mathrm{B}$ corresponded to six doses of nitrogen $(0,60,120,180$, 240 and $300 \mathrm{~kg} \mathrm{ha}^{-1}$ ). Chlorophyll a, b, and total chlorophyll content was evaluated in young and healthy leaves eight months after transplanting.

Results and conclusions: The results showed that the contents of chlorophyll $a, b$ and total were significantly affected by solar radiation, but not by the nitrogen supplied. In both radiation environments, the contents of chlorophyll a were higher than the contents of chlorophyll $b$ in all plants evaluated. In the environment of lower solar radiation (300 $\mu \mathrm{mol}$ of photons $\mathrm{m}^{-2} \mathrm{~s}^{-1}$ ), the maximum levels of the photosynthetic pigments were presented. In the environment of $1.500 \mu \mathrm{mol}$ of photons. $\mathrm{m}^{-2} \mathrm{~s}^{-1}$, the contents of chlorophyll $a, b$ and total were lower in control plants ( 0 $\mathrm{kg} \mathrm{ha}^{-1}$ ).

Keywords: chlorophyll a; chlorophyll b; total chlorophyll; nitrogen; solar radiation 


\section{RESUMEN GRÁFICO}

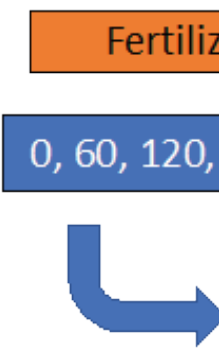

Fertilización nitrogenada

\&

80,240 y $300 \mathrm{~kg} \mathrm{ha}^{-1}$

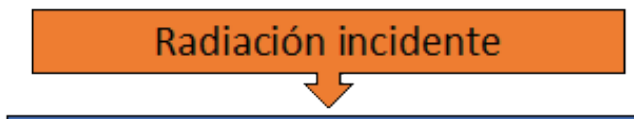

300 y $1500 \mu \mathrm{mol}$ de fotones. $\mathrm{m}^{-2} \mathrm{~s}^{-1}$

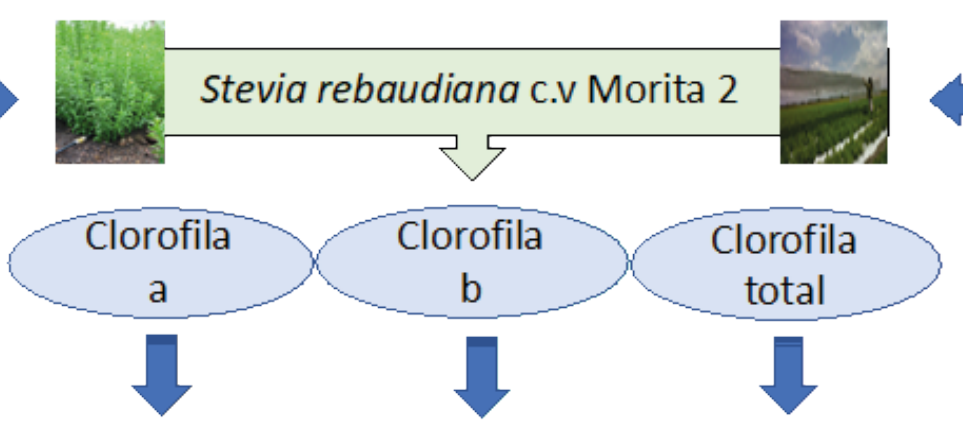

$\checkmark$ Fertilización nitrogenada: ningún efecto

$\checkmark$ Radiación: $\quad$ En ambos ambientes, Clorofila a $(\mathrm{Cl} a)$ fue superior a Clorofila b $(\mathrm{Cl} b)$. $300 \mu \mathrm{mol}$ de fotones. $\mathrm{m}^{-2} \mathrm{~s}^{-1}$, máximos contenidos de $\mathrm{Cl} a, \mathrm{Cl} b$ y total.

Fuente: Autores.

\section{INTRODUCCIÓN}

Los cambios en la composición atmosférica exponen a los seres vivos a nuevas intensidades y longitudes de onda de radiación solar, obligándoles a generar adaptaciones que les permitan sobrellevar estos cambios ambientales (Meisel et al., 2011). La radiación modifica la estructura y función del hábitat de los ecosistemas terrestres y acuáticos, afectando procesos fotobiológicos (fotosíntesis, fotoperiodo, fototropismos), y con repercusiones sobre los factores ambientales (temperatura, humedad relativa, precipitación, brillo solar, nubosidad) y los ciclos naturales (diarios, anuales, hídricos, nutrientes) que finalmente inciden en la distribución y adaptación de los organismos vivos (Haussermann \& Gorsterra, 2009).

La radiación que llega a la tierra abarca una amplia gama del espectro electromagnético y aproximadamente el $40 \%$ de ella es la que conocemos como luz o radiación visible. Esta comprende longitudes de onda que van de los 400 a los $700 \mathrm{~nm}$ y abarca los colores violeta, azul, verde, amarillo, naranja y rojo; al ser usado por los vegetales en el proceso de la fotosíntesis, también se le denomina radiación fotosintéticamente activa o PAR (sigla derivada del inglés Photosynthetic Active Radiation) (Carrasco-Ríos, 2009).

Las plantas, al no estar en capacidad de soportar altas intensidades de luz, principalmente al medio día desarrollaron interesantes adaptaciones, permitiéndoles percibir la luz en distintas longitudes de onda e intensidades, generando respuestas celulares que se traducen en procesos fisiológicos como la reorientación del crecimiento y desarrollo de las especies vegetales (Casal, 2013).

Se ha podido evidenciar que el fototropismo y la reorientación de cloroplastos intracelularmente, al igual que la síntesis de clorofila, son procesos fisiológicos regulados por la luz azul, siendo percibida por la planta debido a la existencia de receptores conocidos como criptocromos y fototropinas (Ballaré, 2014).

La intensidad de la radiación solar y la concentración de nitrógeno en las células vegetales influyen en la síntesis de los pigmentos fotosintéticos. De acuerdo con Khavari-Nejad et al. (2009), una deficiencia 
de nitrógeno, además de acelerar la senescencia foliar y estimular la producción de especies reactivas de oxígeno, conduciría a la degradación de las macromoléculas como proteínas, y por supuesto a la degradación de la clorofila en las plantas. Tal disminución gradual de pigmentos fotosintéticos podría deberse a procesos degenerativos del metabolismo de los cloroplastos, reduciendo así la disponibilidad de clorofila e iniciándose este proceso en las hojas más viejas, debido a la alta movilidad del nitrógeno a través del floema (Franklin \& Quail, 2010; Kong \& Okajima, 2016).

De acuerdo a lo anteriormente expuesto, es importante ampliar la disponibilidad de información actualizada referente a las adaptaciones $\mathrm{y} / \mathrm{o}$ estrategias fisiológicas de las plantas, en particular las de estevia por el interés comercial que tiene, analizando la incidencia positiva en la eficiencia de la producción de clorofila en el proceso de la fotosíntesis. Este análisis funcionaría como una estrategia eficiente que permitirían a los agricultores obtener y mantener los rendimientos estimados en los cultivos (Benzing, 2000).

Estevia es una planta herbácea perenne de la familia Asterácea (que incluye plantas tan conocidas como el diente de león, el girasol, la achicoria y el crisantemo). Las hojas son lanceoladas, tienen aproximadamente $5 \mathrm{~cm}$ de longitud y $2 \mathrm{~cm}$ de ancho, son alternas y enfrentadas en grupo de dos; en forma silvestre alcanza una altura variable entre 40 y $80 \mathrm{~cm}$ y en monocultivo alcanza 1,0 $\mathrm{m}$ de altura (Jarma-Orozco, 2008). Puede utilizarse para la producción comercial por un periodo de cinco o más años, dando varias cosechas anuales a partir de la parte aérea de la planta. Las raíces permanecen en el suelo y permiten el rebrote de la planta, aunque no las macollas. Las plantas con un metro de altura tienen un peso seco medio de $70 \mathrm{~g} \mathrm{y}$ el peso seco de las hojas puede variar entre 15 y 35 g/planta (Jarma et al., 2005).

Teniendo en cuenta la importancia de la estevia actualmente, los objetivos principales de este estudio fueron: a) Determinar la adaptabilidad de los pigmentos fotosintéticos de la estevia a dos niveles de radiación solar incidente $\left(300\right.$ y $1.500 \mu \mathrm{mol}$ de fotones. $\mathrm{m}^{-2}$ $\mathrm{s}^{-1}$ y seis dosis de nitrógeno $(0,60,120,180$, 240 y $300 \mathrm{~kg} \mathrm{ha}^{-1}$ ) y b) Evaluar el contenido de clorofilas $a, b$ y total en hojas jóvenes y sanas a los ocho meses después de trasplante (DDT). Los datos obtenidos serán relevantes para analizar el comportamiento de la estevia frente a los cambios actuales y futuros del ambiente (principalmente a los aumentos en la intensidad de la radiación solar) asociados a fenómenos antropogénicos como el cambio climático.

\section{MATERIALES Y MÉTODOS Localización y material experimental}

La investigación se realizó en el invernadero de la Facultad de Ciencias Agrícolas de la Universidad de Córdoba en el municipio de Montería, departamento de Córdoba (80 $52^{`} \mathrm{LN}$ y $\left.76^{\circ} 58^{`} \mathrm{LO}\right)$ temperatura promedio de $28{ }^{\circ} \mathrm{C}$, humedad relativa promedio del $83 \%$, altura sobre el nivel del mar de 18 msnm y precipitación anual de 1.200 mm. Esta localidad se encuentra en la zona de transición entre Bosque Seco Tropical (BsT) y Bosque Húmedo Tropical (BhT), según la clasificación de Holdridge (1982). El ensayo se estableció mediante un diseño estadístico completamente al azar (DCA) con un arreglo factorial $2 \times 6$, para un total de 12 tratamientos y 16 repeticiones por tratamiento, en el que el factor A correspondió a los dos niveles de radiación solar (300 y $1.500 \mu \mathrm{mol}$ de fotones. $\mathrm{m}^{-2} \mathrm{~s}^{-1}$ correspondien.tes al $20 \%$ y $100 \%$ de radiación respectivamente) y el factor $\mathrm{B}$ correspondió a las seis dosis de nitrógeno ( 0 , $60,120,180,240$ y $300 \mathrm{~kg} \mathrm{ha}^{-1}$ ).

El sustrato utilizado para el semillero (arena lavada) se desinfectó con Metalaxil + Mancozeb, por medio de una mezcla de 30 gramos del producto por cada 10 litros de agua. Luego se sembraron esquejes de estevia con cinco yemas en promedio del genotipo Morita 2. Se utilizó una malla polisombra negra de polietileno de alta densidad para permitir el paso del $20 \%$ de radiación solar incidente, y un ambiente sin polisombra que representó el $100 \%$ de la radiación solar incidente. En total se utilizaron 192 macetas con capacidad de 5 litros cada una, de las cuales 96 correspondieron al ambiente de sombra (20\% de radiación incidente) y 96 al ambiente con el $100 \%$ de la radiación incidente (300 y $1500 \mu \mathrm{mol}$ de fotones. $\mathrm{m}^{-2}$ $\mathrm{s}^{-1}$ respectivamente). 
Cada nivel de fertilización se aplicó en 16 macetas de cada nivel de radiación. Para las soluciones nutritivas se utilizaron las fórmulas del elemento faltante de la solución de Hoagland modificada en agua destilada. El volumen de solución para cada aplicación por planta fue de $200 \mathrm{ml}$. Para el cálculo de las cantidades de nitrógeno necesarias se tuvo en cuenta una densidad de población de 125.000 plantas ha-1 ${ }^{-1}$ Espitia et al. 2009).

Las soluciones se aplicaron cada 4 días después del trasplante. Las respuestas fisiológicas se midieron en función del contenido de pigmentos fotosintéticos (Clorofilas a, b y total), evaluadas en tres plantas por tratamiento, para ello se colectaron hojas del tercio superior alrededor de los ocho meses después del trasplante. Los esquejes provinieron de plantas vigorosas y sanas, sin presencia de flores o botón floral, con 8 y 10 $\mathrm{cm}$ de longitud y como mínimo 5 pares de hojas.

\section{Extracción de clorofila (Clorofilas a, b y total)}

Se tomaron 3 plantas por dosis de nitrógeno, en cada nivel de radiación solar. De estas plantas se tomaron las hojas del tercio superior para realizar el respectivo procedimiento de extracción de pigmentos. La extracción de los pigmentos fotosintéticos se realizó alrededor de los 8 meses después del trasplante (fase vegetativa), es decir, al final del ensayo. Se realizaron las lecturas de la absorbancia a los 470, 645, 652, 662 y $663 \mathrm{~nm}$ con el lector de absorbancia y/o espectrómetro de absorción molecular (Perkin-Elmer Lambda XLS). El proceso de extracción se realizó en el laboratorio de fisiología vegetal de la Universidad de Córdoba y las respectivas lecturas de absorbancia, en el laboratorio de suelos de la Universidad de Córdoba.

Cabe resaltar que para Morita 2 (especie de estudio de esta investigación), la floración inicia aproximadamente a los 90 días después del trasplante. No obstante, en la presente investigación las plantas, durante todo el período evaluado, se mantuvieron en fase vegetativa, ya que las estructuras florales se retiraban de manera permanente en la medida que aparecían (la poda de floración es una labor contemplada para un mejor manejo de la plantación). Para la extracción y determinación de clorofilas $a$ y $b$ presentes en hojas de estevia se utilizó acetona al $100 \%$ y se siguió la metodología descrita a continuación:

1. Se colocó en el mortero $1 \mathrm{~g}$ de hojas de estevia, sin las nervaduras grandes y cortadas en pequeños tamaños.

2. Se agregó arena y $4 \mathrm{ml}$ de acetona al mortero para moler el tejido y obtener una pasta fina. Se adicionaron $20 \mathrm{ml}$ más de acetona.

3. Se transfirió cuidadosamente el extracto resultante al embudo de Buchner provisto de papel filtro, y se filtró al vacío.

4. Se agregaron otros $60 \mathrm{ml}$ de acetona a la pulpa de las hojas y se reanudó la molienda y el filtrado. Este segundo extracto se agregó al primero.

5. Después de lavar el mortero y el embudo con $50 \mathrm{ml}$ de acetona, este contenido se incorporó al filtrado.

6. Se realizó la lectura de la densidad óptica o absorbancia (D) del extracto a $470,645,652,662$ y $663 \mathrm{~nm}$.

Las ecuaciones que se utilizaron para los cálculos de los pigmentos fotosintéticos (Clorofila a ( $\mathrm{Cl}$ a); Clorofila b (Cl b) y Clorofila total $(\mathrm{Cl} \mathrm{t})$ son las siguientes:

Ecuación 1

$\mathrm{Cl}$ a $(\mu \mathrm{g} \cdot \mathrm{ml}-1)=10,81$ Abs662 - 0,75 Abs645

Ecuación 2

Cl b $(\mu \mathrm{g} \cdot \mathrm{ml}-1)=19,02$ Abs645 - 3,98 Abs662

Ecuación 3

$\mathrm{Cl}$ t $(\mu \mathrm{g} \cdot \mathrm{ml}-1)=6,83$ Abs662 $+18,27$ Abs645

La concentración de clorofila fue expresada en $\mu \mathrm{g}$ por $\mathrm{ml}$ de acetona al $100 \%$ (Val et al. 1985).

\section{Análisis estadísticos}

Los resultados se analizaron mediante el paquete Statistical Analysis System (SAS) versión 9.1, con licencia para la Universidad 
de Córdoba. Previamente al análisis de la varianza, se verificó la normalidad de los datos a través de la prueba de Shapiro-Wilks. Las pruebas de comparación de medias se utilizaron cuando se detectaron diferencias estadísticas en los factores individuales mediante la prueba de Tukey, con nivel de probabilidad del $5 \%$.

\section{RESULTADOS Y DISCUSIÓN CLOROFILAS}

Los resultados más relevantes indicaron que solamente el efecto de la radiación tuvo un efecto significativo $(P<0,01)$ sobre las concentraciones de las clorofilas $\mathrm{a}, \mathrm{b}$ y total (Tabla 1).

Tabla 1. Cuadrados medios y niveles de significancia para las variables Clorofilas a, b y Clorofila total de estevia, en función de dos niveles de radiación solar y seis dosis de nitrógeno.

$\begin{array}{lrrrr}\begin{array}{c}\text { Fuente de } \\ \text { variación }\end{array} & \text { GL } & \begin{array}{r}\text { Clorofilla a } \\ \left(\mu g \mathrm{ml}^{-1}\right)\end{array} & \begin{array}{r}\text { Clorofilla b } \\ \left(\mu g \mathrm{ml}^{-1}\right)\end{array} & \begin{array}{r}\text { Clorofilla total } \\ \left(\mu \mathrm{ml}^{-1}\right)\end{array} \\ \text { Radiación solar } & 1 & 276,113^{* *} & 654,506 * * & 1783,654 * * \\ \text { Nitrógeno } & 5 & 0,997 \mathrm{NS} & 0,490 \mathrm{NS} & 1,295 \mathrm{NS} \\ \text { Radiación Nitrógeno } & 5 & 2,533 \mathrm{NS} & 3,268 \mathrm{NS} & 10,887 \mathrm{NS} \\ \text { Error } & 24 & 4,429 & 4,499 & 15,634 \\ \text { R2 } & & 0,73 & 0,86 & 0,83 \\ \text { CV } & 11,64 & 18,76 & 13,45\end{array}$

**: Significativo al 1\%. NS: No significativo.

\section{Fuente: autores}

En la Tabla 2 se muestran los valores promedio de la producción de clorofilas $a, b$ y total. La concentración de clorofila a osciló entre 20,43 y $21,46 \mathrm{\mu g} \mathrm{ml}^{-1}$ para la radiación de $300 \mu \mathrm{mol}$ de fotones $\mathrm{m}^{-2} \mathrm{~s}^{-1}$, y entre 13,84 y16,89 $\mu \mathrm{gml}-1$ para la radiación de $1.500 \mu \mathrm{mol}$ de fotones $\mathrm{m}^{-2} \mathrm{~s}^{-1}$. Es importante resaltar que en el ambiente de baja radiación ( $300 \mu \mathrm{mol}$ de fotones $\mathrm{m}^{-2} \mathrm{~s}^{-1}$ ) se presentó una producción $27 \%$ mayor de $\mathrm{Cl}$ a, superando significativamente $\quad(p<0,05)$ los niveles observados en el ambiente alta radiación.

La concentración de $\mathrm{Cl}$ b registró un comportamiento similar al de la $\mathrm{Cl}$ a. Los valores de clorofila b oscilaron entre 14,17 y $16,56 \mu \mathrm{g} \mathrm{ml}^{-1}$ para $300 \mu \mathrm{mol}$ de fotones $\mathrm{m}^{-2} \mathrm{~s}^{-1}$, y entre 6,15 y 7,92 $\mu \mathrm{g} \mathrm{ml}-1$ para $1500 \mu \mathrm{mol}$ de fotones $\mathrm{m}^{-2} \mathrm{~s}^{-1}$. La prueba de comparación de medias, entre los ambientes de radiación, indicó que en ambientes de baja radiación se registra aproximadamente un $55 \%$ de mayor contenido promedio de clorofila $b$ respecto $a$ un ambiente de alta radiación.

De manera consistente con los resultados observados para las $\mathrm{Cl}$ a y $\mathrm{Cl}$ b, la clorofila total presentó respuestas diferenciales significativas $(p<0,05)$ solo por el efecto de la radiación solar, oscilando entre 34,60 y $37,72 \mu \mathrm{g} \mathrm{ml}^{-1}$ para el nivel de radiación de $300 \mu \mathrm{mol}$ de fotones $\mathrm{m}^{-2} \mathrm{~s}^{-1}$, y entre 19,99 y $24,81 \mu \mathrm{g} \mathrm{ml}^{-1}$ para el de $1.500 \mu \mathrm{mol}$ de fotones $\mathrm{m}^{-2} \mathrm{~s}^{-1}$ (Tabla 2).

Tabla 2. Prueba de comparación de medias (Tukey) para las variables Clorofilas a, b y total de Stevia rebaudiana Bert. en función de la Radiación solar.

\begin{tabular}{|c|c|c|c|}
\hline Radiación solar & $\begin{array}{c}\text { Clorofilla a } \\
\left(\mu g \mathrm{~m}^{-1}\right)\end{array}$ & $\begin{array}{c}\text { Clorofilla b } \\
\left(\left.\mu g\right|^{-1}\right)\end{array}$ & $\begin{array}{l}\text { Clorofila total } \\
\left(\mu \mathrm{ml}^{-1}\right)\end{array}$ \\
\hline $300 \mu \mathrm{mol}$ de fótones. $\mathrm{m}-2 \mathrm{~s}-1$ & 20,85 a & 15,56 a & $36,43 a$ \\
\hline $1.500 \mu \mathrm{mol}$ de fótones. $\mathrm{m}-2 \mathrm{~s}-1$ & $15,31 b$ & $7,039 b$ & $22,35 b$ \\
\hline
\end{tabular}


Al analizar la prueba de comparación entre medias para determinar el efecto de la radiación solar sobre la concentración de la clorofila total, se pudo establecer que a un nivel menor de radiación se presentó alrededor de 1,6 veces mayor contenido promedio de $\mathrm{Cl}$ total, respecto al nivel de alta radiación incidente.

Con relación a los resultados, hay que decir que el aumento de los contenidos de clorofila en las plantas que crecen en ambientes de poca radiación solar constituye una estrategia fisiológica, que le permite a las mismas garantizar una óptima captación de luz; de tal manera que la cantidad de energía percibida permita excitar las moléculas de la proteína necesaria para iniciar el proceso fotoquímico y así sintetizar poder energético y reductor, necesarios para la asimilación de $\mathrm{CO}_{2}$ en estos ambientes de sombra. La mayoría de los trabajos que evalúan el efecto de la radiación sobre la concentración de los pigmentos fotosintéticos confirman lo expresado. Campanello et al. (2011), manifiestan que individuos juveniles de especies menos tolerantes a la sombra pueden responder rápidamente a los cambios en los niveles de radiación, ajustando su fisiología y morfología para mantener tasas de crecimiento mayores que las de especies más tolerantes.

Así mismo, Martin et al. (1999), indican que hojas de Bromelia karatas presentaron respuestas típicas de aclimatación a sol y sombra, con mayores concentraciones de clorofila total en plantas de sombra, comparada con plantas expuestas a radiación directa. Los valores de clorofilas totales fueron similares y comparables con los encontrados en otras bromeliáceas terrestres (Benzing, 2000; Skillman et al., 2005; Matsubara et al., 2009).

Estos resultados confirmarían los reportados para la estevia en este estudio. Otros estudios también corroboran los hallazgos reportados en este estudio con estevia. Por ejemplo, Santelices et al. (2013) reportan que las plantas de Nothofagus leonii sometidas a mayor sombra presentaron mayor cantidad de clorofila que las plantas a plena luminosidad, lo que a su vez coincide con lo reportado por Taiz \& Zeiger (2012).
Por su parte, autores como Elorza et al. (2007), al trabajar en Vanilla planifolia Andrews, también observaron que el mayor contenido de clorofila se produjo en plantas bajo los sistemas de malla sombra. Carrasco y Escobar (2002), de acuerdo con sus resultados, reportaron que las plantas en condiciones de un $80 \%$ de sombra contienen una mayor cantidad de pigmentos foliares, tanto de clorofila a como de $b$, lo que se expresó en el color verde más oscuro de las hojas. Los valores corresponden a 10,29 y $4,78 \mathrm{mg} \mathrm{g}^{-1}$ de peso fresco para clorofila a y b con $80 \%$ de sombra y de 5,32 y 2,37 para clorofila a y b sin sombra. Un comportamiento similar fue reportado por Arboleda (2011) en Aptenia cordifolia. Estos resultados condicen con los encontrados para estevia en este estudio.

Aunque los resultados de esta investigación coinciden con los de otras especies, tal como se mencionó anteriormente y como lo reportan Fahl et al. (1994); Carelli y Fahl (2000); Pompelli (2008), quienes encontraron en plantas jóvenes de ciertos cultivares de café que los contenidos de clorofila $a, b$ y total se incrementaron en las plantas crecidas a la sombra, en comparación con las expuestas a pleno sol.

Trabajos como los de Del Ángel-Hernández et al. (2017) en una investigación en calabacita (Cucurbita pepo cv. Zucchini), reportaron que plantas que crecieron bajo un túnel con policarbonato de color claro (el de mayor transmitancia 82,57\%), tuvieron mayor contenido de clorofila y mayor desarrollo foliar. Por el contrario, las plantas en un túnel de policarbonato rojo (que tuvo la menor transmitancia, 23,51\%), que recibieron la menor cantidad de radiación PAR, presentaron menor contenido de clorofila y menor desarrollo foliar.

Para el caso de la estevia en el presente estudio, la mayor producción de $\mathrm{Cl}$ a y $\mathrm{Cl}$ b (a $300 \mu \mathrm{mol}$ de fotones $\mathrm{m}^{-2} \mathrm{~s}^{-1}$ ) pudo manifestarse como una estrategia propia de las plantas de sombra para tratar de crecer y desarrollarse bajo condiciones de poca luz; sin embargo, esta estrategia no se vio reflejada en la producción de biomasa y en las tasas de asimilación de $\mathrm{CO}_{2}$, que fueron 
significativamente menores en un ambiente de baja radiación respecto a uno de alta radiación (datos no mostrados). Es importante aclarar que en condiciones de alta radiación, como las que regularmente se registran en el Caribe colombiano (Burbano-Erazo et al. (2020), los pigmentos que (en su orden) procesan mayor cantidad de energía radiante son los carotenos, las clorofilas b y por último las clorofilas a (Taiz \& Zeiger, 2012).

Consecuentemente con lo anterior, sería de esperarse que los niveles de $\mathrm{Cl} b$ registren un aumento respecto a los de $\mathrm{Cl}$ a cuando la radiación es mayor; sin embargo, es probable que en este trabajo no se detecten esas diferencias, y que la condición de adaptación de la estevia a la alta radiación se vea reflejada en el aumento en la producción de carotenos, los cuales desafortunadamente no se midieron.

Otras condiciones estresantes, que pueden derivarse de una foto-saturación lumínica, pueden también tener un efecto en el decrecimiento de clorofilas en la planta. González-Salvatierra et al. (2013) manifiestan que durante la temporada de sequía, como respuesta al exceso de luz, los tejidos de las hojas de plantas expuestas y de sombra de Bromelia karatas disminuyeron el contenido de clorofilas e incrementaron el contenido de carotenoides. Por su parte, los estudios realizados por Peñaranda et al. (2020) en Baccharis latifolia encontraron que la concentración de clorofilas se vio favorecida por los tratamientos de PAR y UVA50 (50\% de UVB respecto a la radiación solar diaria), y fue negativamente influenciada por los tratamientos UVB100 y UVB150.

Respecto a la relación de clorofilas a y b, Casierra-Posada et al. (2012) al estudiar parámetros fisiológicos de la caléndula y evaluar cada hora el contenido de clorofilas y carotenos en plantas cultivadas a plena exposición, bajo una malla de polisombra con $37 \%$ de reducción de luz, encontraron que los contenidos de clorofila b y clorofila total mostraron diferencias a lo largo del día, con tendencia al incremento entre las 6:00 y 18:00 horas; además, el valor de la relación clorofila a-clorofila b fue más elevado en las hojas de plantas sombreadas y el valor de la relación carotenos/clorofila fue más alto en las plantas que crecieron a plena exposición.
En el caso de la estevia, esta relación no fue evidente al compararse con los resultados de Casierra-Posada et al. (2012), lo que podría indicar que la respuesta de reducción de clorofilas en ambientes de alta radiación fue igual para ambas. La discusión la complementa Reddy (2006), quien menciona que la disminución gradual de los pigmentos fotosintéticos y la clorosis podrían deberse a procesos degenerativos del metabolismo de los cloroplastos, reduciendo así la disponibilidad de clorofila. Este proceso se inicia en las hojas más viejas, debido a la alta movilidad del nitrógeno a través del floema. Esto coincide con lo reportado por Khavari-Nejad et al. (2009), quienes afirman que la deficiencia de nitrógeno acelera la senescencia foliar y estimula la producción de especies reactivas de oxígeno, lo que conduce a la degradación de las macromoléculas como proteínas y la clorofila en las plantas.

Los resultados obtenidos para las plantas de Stevia expuestas al $100 \%$ de radiación solar no coinciden con los reportados por Chaves (2007) al estudiar el retraso del reverdecimiento en las hojas nuevas de Pernettya prostrata (Ericaceae), pues sus resultados sugieren que la pigmentación rojiza de las hojas nuevas de esta especie vegetal podría representar una adaptación fisiológica contra el exceso de radiación solar que estas reciben en ambientes de páramo. Así mismo, Aguirre (2009) encontró que en condiciones de invernadero, las hojas de Solanum viarum (Solanaceae) que crecieron bajo sombra presentaron menor contenido de clorofila. De igual forma, los resultados obtenidos en este estudio difieren de los resultados expuestos por Ramos et al. (2013), quienes en un trabajo con pasturas observaron que a la mayor temperatura y a la mayor radiación se evidenció un aumento del contenido de clorofilas de Polylepis cuadrijuga, evidenciado por la presencia de un parénquima en empalizada estratificado.

\section{NITRÓGENO}

Aunque en esta investigación no se evidenció un efecto estadísticamente significativo del nitrógeno sobre la producción de pigmentos fotosintéticos en estevia, Utumi et al. (1999) reportaron que plantas 
de esta especie vegetal con deficiencia de nitrógeno mostraron reducción generalizada del crecimiento, observadas en ramificaciones delgadas y alargadas, presentando disminución en el contenido de clorofila en las hojas basales. Lo anterior probablemente podría ser consecuencia de una baja producción de clorofilas, lo que afectaría de manera importante las tasas de fotosíntesis y, por lo tanto, tendría un efecto negativo sobre el crecimiento.

En la Figura 1 ( $a$ y b), se comparan los contenidos de los pigmentos fotosintéticos de estevia (clorofilas a, b y total) para cada ambiente y nivel de fertilización nitrogenada.

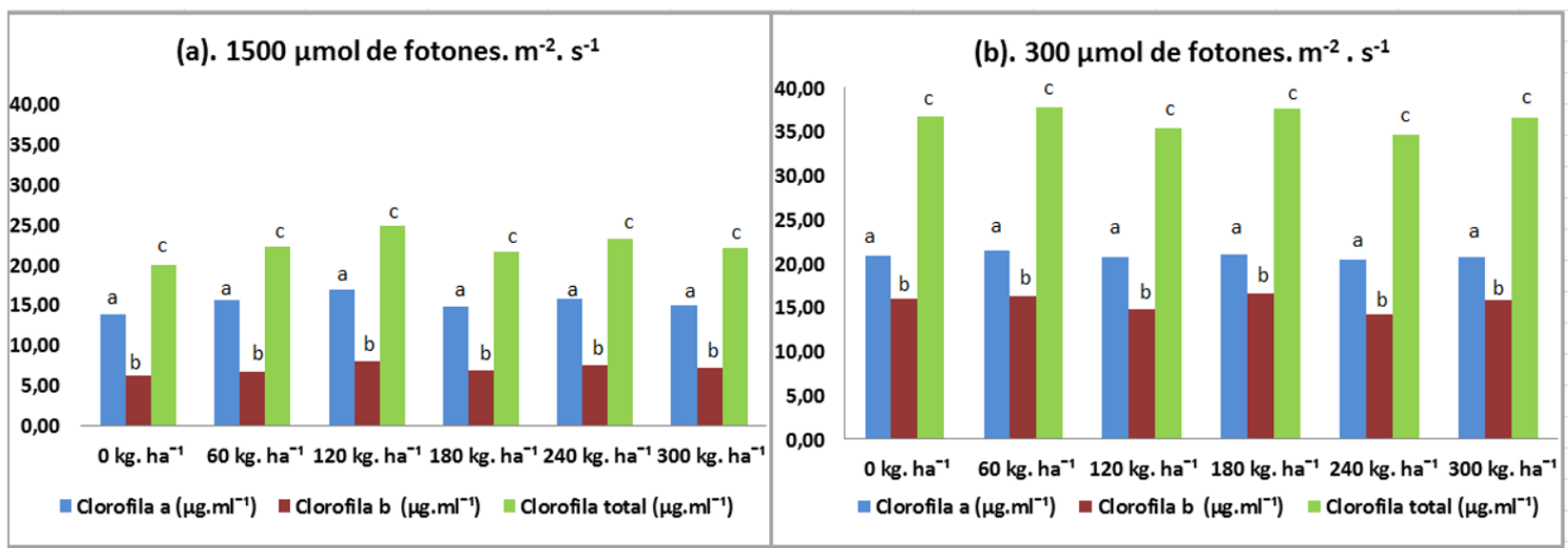

Figura 1 a y b. Contenidos de clorofila a, b y total en función de la fertilización nitrogenada en Stevia Rebaudiana Bert. a los 8 meses después del trasplante, en plantas sometidas a 1.500 (a) y $300 \mu \mathrm{mol}$ de fotones. $\mathrm{m}^{-2} \mathrm{~s}^{-1}$ (b). Los promedios con la misma letra, dentro de un mismo nivel de radiación, no difieren estadísticamente.

Fuente: autores

Como se puede observar, hay una evidencia clara de que, independientemente de la dosis de nitrógeno, en ambos ambientes los contenidos de clorofila a fueron mayores en todas las plantas evaluadas, comparándolos con los contenidos de clorofila $b$ (Figura 1 a y b).

A pesar de no encontrar una relación directa con los niveles de nitrógeno en este estudio, es importante considerar otras investigaciones que se han realizado con relación al efecto del nitrógeno y la producción de clorofilas en otras especies. Gutiérrez Del Pozo (2010) afirma que el aumento del suministro de nitrógeno incrementa la concentración de clorofila y no altera el balance entre la clorofila y la rubisco o el nitrógeno total en las hojas relativamente jóvenes.

Autores, como Mendoza-Elos et al. (2006), reportan que los niveles más altos de clorofila en los maíces, de alta calidad proteínica (QPM) y normal $V_{1}$ blanco, se obtuvieron cuando las plantas se fertilizaron con 100 y $200 \mathrm{~kg} \mathrm{ha}^{-1}$ de nitrógeno. Este resultado no coincide con los encontrados en este ensayo, en el cual, utilizando dosis de nitrógeno cercanas
(60 y $120 \mathrm{~kg} \mathrm{ha}^{-1}$ ), no se presentó aumento estadísticamente significativo del contenido de clorofila.

En lo referente a la evaluación del contenido de clorofila a través de métodos no destructivos, es decir utilizando equipos como el SPAD, Salgado (2013) encontró para estevia que el tratamiento con $9 \mathrm{meq} \mathrm{L}^{-1} \mathrm{de} \mathrm{NO}_{3}^{-}$originó los mayores valores en las respuestas de las variables fisiológicas, incluyendo las lecturas SPAD que normalmente se usan para medir contenido de clorofilas (Soil Plant Analysis Development). Por otra parte, aunque estos resultados no coinciden con lo reportado estadísticamente en este estudio, Medina (2011), reportó que la reducción de nitrógeno provocó una disminución significativa en la concentración total de clorofila en brotes de Castilleja tenuiflora.

\section{Curvas de absorbancia de pigmentos fotosintéticos de Stevia rebaudiana Bert.}

En la Figura 2 ( $a$ y b), se muestran las curvas de absorbancia de pigmentos fotosintéticos de estevia a los 8 meses después del trasplante. 


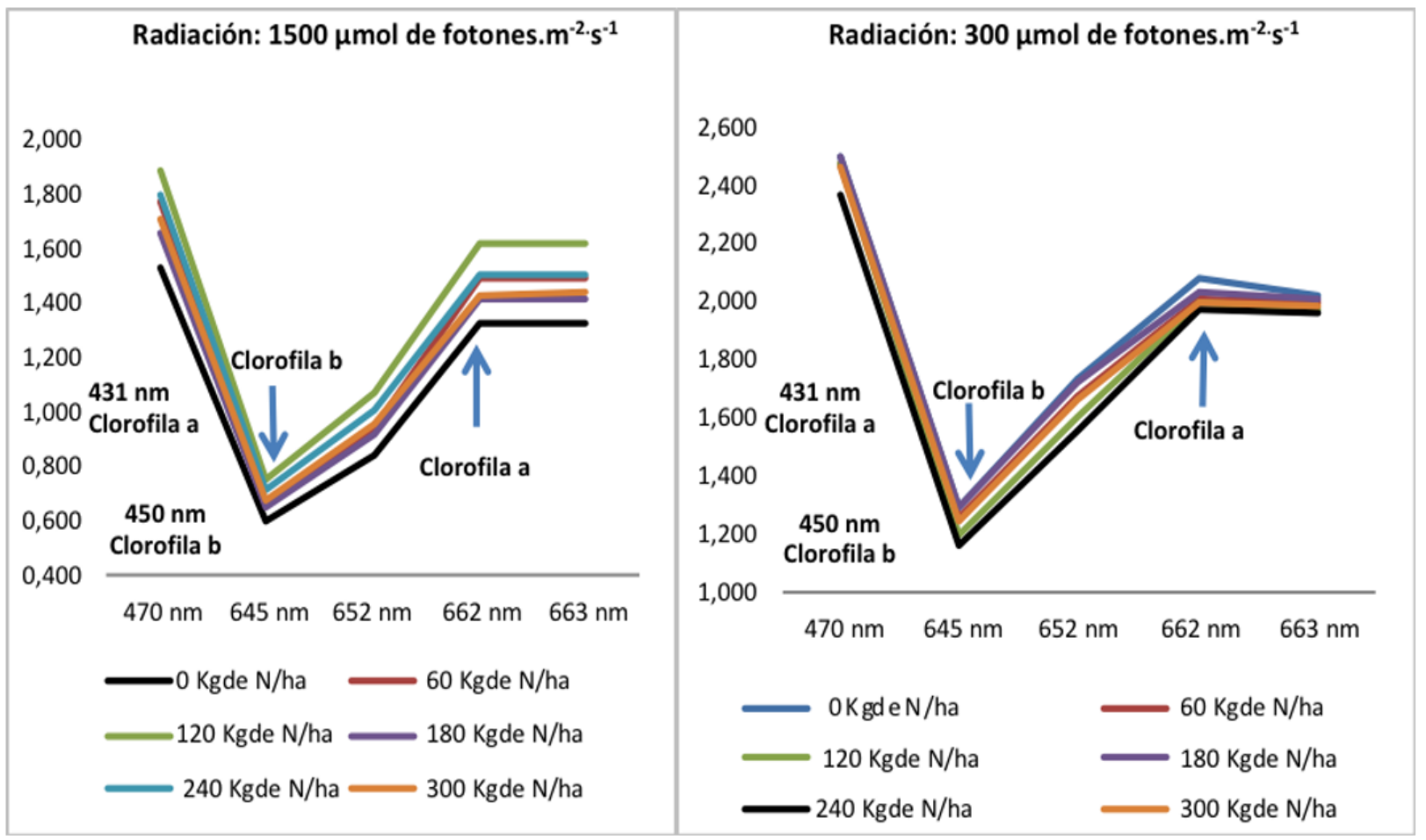

Figura 2. Curvas de absorbancia de pigmentos de Stevia Rebaudiana Bert. Los pigmentos se extrajeron con acetona al $100 \%$, a los 8 meses después del trasplante, en un ambiente de 1.500 (a) y $300 \mu \mathrm{mol}$ de fotones. $\mathrm{m}^{-2} \mathrm{~s}^{-1}$ (b).

Fuente: autores

Los máximos valores de clorofilas $a$ y $b$, usando acetona al $100 \%$, aparecen a los 662 y $645 \mathrm{~nm}$ respectivamente. También estos pigmentos presentan absorción a $431 \mathrm{~nm}$ para la clorofila a y a $450 \mathrm{~nm}$ para la clorofila b (Val et al., 1985). Estos resultados son similares a los reportados por Medina (2011) quien determinó los contenidos de clorofila en brotes de Castilleja tenuiflora, utilizando el método de Lichtenthaler \& Wellburn (1983), haciendo las lecturas de los pigmentos fotosintéticos en absorbancias similares.

\section{CONCLUSIONES}

Los contenidos de clorofilas $a$, b y total en estevia fueron afectados significativamente por la radiación solar. En ambos ambientes de luz, los contenidos de clorofila a fueron superiores en todas las plantas evaluadas, al compararse con los contenidos de clorofila b. En el ambiente de menor radiación solar ( $300 \mu \mathrm{mol}$ de fotones $\mathrm{m}^{-2} \mathrm{~s}^{-1}$ ), se presentaron los máximos contenidos de los pigmentos fotosintéticos. Aunque este ensayo no evidenció un efecto significativo de las dosis de nitrógeno, ni de la interacción radiación solar-nitrógeno, sobre el comportamiento de los pigmentos fotosintéticos de estevia, se observó que, a diferencia del ambiente de $300 \mu \mathrm{mol}$ de fotones. $\mathrm{m}^{-2} \mathrm{~s}^{-1}$, en el que los valores de los pigmentos no presentaron una tendencia definida con respecto a las dosis de nitrógeno aplicadas, en el ambiente de 1500 umol de fotones. $\mathrm{m}^{-2} \mathrm{~s}^{-1}$, los contenidos de clorofilas a, b y total fueron menores en las plantas testigo $\left(0 \mathrm{~kg} \mathrm{ha}^{-1}\right)$.

Para el caso de la estevia en el presente estudio, aunque se resalta nuevamente que es una planta heliófila, el comportamiento del genotipo evaluado Morita 2 (a $300 \mu \mathrm{mol}$ de fotones) $\mathrm{m}^{-2} \mathrm{~s}^{-1}$ pudo manifestarse como una estrategia típica de las plantas de sombra para tratar de crecer y desarrollarse bajo condiciones de poca luz, aumentando los contenidos de clorofilas a, b y total, pero con menor cantidad de biomasa. 


\section{RECOMENDACIONES}

Atendiendo a que la molécula de clorofila está compuesta por los elementos nitrógeno y magnesio, para futuros ensayos se recomienda evaluar el efecto de ambos macroelementos de manera simultánea y así corroborar la presencia o ausencia de un efecto significativo sobre la producción de pigmentos fotosintéticos en las plantas de estevia.

\section{CONTRIBUCIÓN DE LA AUTORÍA}

Primer autor: adquisición de recursos, administrador del proyecto, análisis de datos, conceptualización y escritura. Segundo autor: metodología, conceptualización, trabajo de campo, investigación, análisis de datos, escritura y borrador original. Tercer autor: conceptualización, logística, revisión, edición, control de estilo y borrador final.

\section{AGRADECIMIENTOS}

Los autores expresan sentidos agradecimientos a la Universidad de Córdoba por el apoyo económico y logístico brindado para la realización de la presente investigación.

\section{LITERATURA CITADA}

Aguirre, C. A. (2009). Efecto de la calidad de la hoja de Solanum viarum (Solanaceae) en el desarrollo de Gratiana boliviana (Coleóptera: Crisomelidae) [Tesis de F pregrado, Universidad Zamorano]. Biblioteca Digital Wilson Popenoe. https://bdigital. zamorano.edu/bitstream/11036/340/1/ T2718.pdf

Arboleda, M. E. (2011). Efecto de la irradiancia en el crecimiento y desarrollo de Aptenia cordifolia (L.f) Schwantes como cobertura ornamental. Bioagro, 23(3), 175184.

Ballaré, C. L. (2014). Light regulation of plant defense. Annual review of plant biology, 65, 335-363. https://doi.org/10.1146/ annurev-arplant-050213-040145

Benzing, D. H. (2000). Bromeliaceae: Profile of an Adaptive Radiation. Cambridge
University Press. https://doi.org/10.1017/ CBO9780511565175

Burbano-Erazo, E., Cordero, C., Pastrana, I., Espitia, L., Gómez, E., Morales, A., Pérez, J., López, L. \& Rosero, A. (2020). Interrelation of Ecophysiological and Morpho-Agronomic Parameters in Low Altitude Evaluation of Selected Ecotypes of Sweet Potato (Ipomoea batatas [L.] Lam.). Horticulturae, 6(4), 99. https://doi. org/10.3390/horticulturae6040099

Campanello, P. I., Gatti, M. G., Montti, L., VIillagra, M. y Goldstein, G. (2011). Ser o no ser tolerante a la sombra: economía de agua y carbono en especies arbóreas del Bosque Atlántico (Misiones, Argentina). Ecología Austral, 21(3), 285-300.

Carelli, M. L. C. y Fahl, J. I. (2000). Crecimiento y asimilación del Carbono y Nitrógeno en plantas jóvenes de Coffea en condiciones de sol y de sombra. En L. Zamora y J. Echeverry. (Eds.). XIX Simposio Latinoamericano de Caficultura: Memorias pp. 101-108. Icafé.

Carrasco, L. y Escobar, H. (2002). Cambios en los contenidos de clorofila, proteínas y niveles de fluorescencia de clorofila en plantas de café (Coffea arabica L.) cultivadas en zonas áridas en diferentes condiciones de luminosidad. Idesia, 20(2), 111-118. https://www.idesia.cl/Numeros/ IDESIA_21/CAP4CA1.PDF

Carrasco-Ríos, L. (2009). Efecto de la radiación ultravioleta-b en plantas. Idesia, 27(3), 59-76.

Casal, J. (2013). Photoreceptor signaling networks in plant responses to shade. Annual review of plant biology, 64, 403-427.

https://doi.org/10.1146/annurevarplant-050312-120221

Casierra-Posada, F., Ávila-León, O. y Riascos-Ortíz, D. (2012). Cambios diarios del contenido de pigmentos fotosintéticos en hojas de caléndula bajo sol y sombra. Temas Agrarios, 17(1), 60-71. 
Chaves, O. (2007). Retraso del enverdecimiento en las hojas nuevas de Pernettya prostrata (ericaceae): posibles funciones adaptativas. Pensamiento actual, 7(8-9), 96-104. https://dialnet.unirioja.es/ descarga/articulo/5897840.pdf

Del Ángel-Hernández, M., ZermeñoGonzález, A., Melendres-Álvarez, A., Campos- Magaña, S. G., Cadena-Zapata, M. y Del Bosque-Villarreal, G. A. (2017). Características de la cubierta de un túnel efecto en radiación, clorofila y rendimiento de calabacita. Revista Mexicana de Ciencias Agrícolas, 8(5), 1127-1142.

Elorza, P., López, M., Hernández, A., Olmedo, G., Domínguez, C. y Maruri, J. (2007). Efecto del tipo de tutor sobre el contenido de vainillina y clorofila en vainas de vainilla (Vanilla planifolia Andrews) en Tuxpan, Veracruz, México. Revista Científica UDO Agrícola, 7(1), 228-236. https://doi. org/10.47808/revistabioagro.v9i1.345

Espitia, M., Montoya, R. y Atencio, L. (2009). Rendimiento de Stevia rebaudiana Bert. bajo tres arreglos poblacionales en el Sinú Medio. Revista U.D.C.A Actualidad \& Divulgación Científica, 12(1), 151-161.

Fahl, J., Carelli, M., Vega, J. \& Magalhaes, A. (1994). Nitrogen and irradiance levels affecting net photosynthesis and growth of young coffee plants (Coffea Arabica L.). Journal of Horticultural Science, 69(1), 161169.

Haussermann, V. \& Gorsterra, G. 2009. Ecological and biogeographical aspects of the Chilean Fjord Region. En V. Haussermann \& G. Gorsterra (Eds). Marine benthic fauna of Chilean Patagonia (pp. 61-76). Nature in Focus.

Franklin, K. A. \& Quail, P. H. (2010). Phytochrome functions in Arabidopsis development. Journal of experimental Botany, 61(1), 11-24. https://doi.org/10.1093/jxb/ erp304

González-Salvatierra, C., Andrade, J. L., Orellana, R., Peña-Rodríguez, L. M. y Reyes-
García, C. (2013). Microambiente lumínico y morfología y fisiología foliar de Bromelia karatas (Bromeliaceae) en una selva baja caducifolia de Yucatán, México. Botanical Sciences, 91(1), 75-84.

Gutiérrez Del Pozo, D. (2010). Aclimatación de la fotosíntesis en el dosel vegetal del trigo al aumento del $\mathrm{CO}_{2}$ atmosférico, función del nitrógeno y las citoquininas en cultivos en cámaras de campo con clima mediterráneo [Tesis Doctoral, Universidad de Salamanca]. Digital. CSIC. https://digital.csic.es/ bitstream/10261/29201/3/DGP-tesis.pdf

Holdridge, L. R. (1982). Ecología basada en zonas de vida. Instituto Interamericano De Cooperación Para La Agricultura. http:// www.cct.or.cr/contenido/wp-content/ uploads/2017/11/Ecologia-Basada-enZonas-de-Vida-Libro-IV.pdf

Jarma, A., Rengifo, T. y AraméndizTatis, H. (2005). Aspectos fisiológicos de estevia (Stevia rebaudiana Bertoni) en el Caribe colombiano: I. Efecto de la radiación incidente sobre el área foliar y la distribución de biomasa. Agronomía Colombiana, 23(2), 207-216. https://www.redalyc.org/ pdf/1803/180316955003.pdf

Jarma-Orozco, A. (2008). Estudios de adaptación y manejo integrado de estevia (Stevia rebaudiana Bert.): nueva alternativa agroindustrial del Caribe colombiano. Una revisión. Revista Colombiana de Ciencias Hortícolas, 2(1), 109-121. https://doi. org/10.17584/rcch.2008v2i1.1176

Khavari-Nejad, R., Najafi, F. \& Tofighi, C. (2009). Diverse responses of tomato to $\mathrm{N}$ and P deficiency. International Journal Agriculture Biology, 11(2), 209-213. http://www. fspublishers.org/published_papers/93814. pdf

Kong, S. \& Okajima, K. (2016) Diverse photoreceptors and light responses in plants. Journal of Plant Research, 129, 111114. https://link.springer.com/content/ pdf/10.1007/s10265-016-0792-5.pdf

Lichtenthaler, H. \& Wellburn, A. (1983). Determination of total carotenoids and 
chlorophylls A and B of leaf in different solvents. Biology Society Transaction, 11 (5), 591-592. https://doi.org/10.1042/ bst0110591

Martin, C. E., Tüffers, A., Herppich, W. B. \& Von Willert, D. J. (1999). Utilization and dissipation of absorbed light energy in the epiphytic crassulacean acid metabolism bromeliad Tillandsia ionantha. International Journal of Plant Sciences, 160(2), 307-313. https://kuscholarworks. ku.edu/bitstream/1808/9892/1/Martin_ IJoPS_160_307-313.pdf

Matsubara, S., Krause, G. H., Aranda, J. E., Virgo, A., Beisel, K. G., Jahns, P. \& Winter, K. (2009). Sun-shade patterns of leaf carotenoid composition in 86 species of neotropical forest plants. Functional Plant Biology, 36(1), 2036. https://doi.org/10.1071/FP08214

Medina, V. (2011). Efecto de la reducción de nitrógeno en el metabolismo de compuestos fenólicos en brotes de Castilleja tenuiflora Benth. cultivados en biorreactor de inmersión temporal [Tesis de Maestría, Instituto politécnico Nacional]. Repositorio Digital IPN. http://repositoriodigital.ipn.mx/ handle/123456789/7283

Meisel, L., Urbina, D. y Pinto, M. (2011). Fotorreceptores y Respuestas de Plantas a Señales Lumínicas. Universidad de La Serena.

Mendoza-Elos, M., Mosqueda-Villagómez, C., Rangel-Lucio, J. A., López-Benítez, A., Rodríguez-Herrera, S. A., Latournerie-Moreno, L. y Moreno-Martínez, E. (2006). Densidad de población y fertilización nitrogenada en la clorofila, materia seca y rendimiento de maíz normal y QPM. Agricultura Técnica en México, 32(1): 89-99. http://www.scielo.org.mx/pdf/ agritm/v32n1/v32n1a9.pdf

Peñaranda, J. C., Rodrigo, G. C., TiconaBustillos, A. R., Valenzuela, E., Ramos, S., San Martin, A., Ghezzi, F. G. y Almanza, G. R. (2020). Variation in concentration of flavonoids and chlorophyll, and changes on morphology and foliar anatomy, due to visible (PAR) or ultraviolet (UVA, UVB) radiation in Baccharis Latifolia. Revista Boliviana de Química, 37(5), 210-222.

Pompelli, M. (2008). Crescimento e adaptações morfofisiológicas de plantas jovens de Coffea arabica L., submetidas a diferentes níveis de nitrogênio e irradiância [Tesis de Doctorado, Universidad Federal de Viçosa].

Ramos, C., Buitrago, S. P., Pulido, K. L. y Vanegas, L. (2013). Variabilidad ambiental y respuestas fisiológicas de Polylepis cuadrijuga (Rosaceae) en un ambiente fragmentado en el Páramo de la Rusia (Colombia). Revista de Biología Tropical, 61(1), 351-361. https:// www.scielo.sa.cr/pdf/rbt/v61n1/a30v61n1. pdf

Reddy, K. (2006). Nutrient Stress. En K. V. Madhava, A. S. Raghavendra \& K. J. Reddy. (Eds.). Physiology and Molecular Biology of Stress Tolerance in Plants (pp. 187-217). Springer.

Salgado, L. (2013). Requerimiento de nitrógeno en la producción de Stevia rebaudina Bertoni en hidroponía en invernadero [Tesis Maestría, Colegio de Postgraduados]. Repositorio institucional. http://hdl.handle. net/10521/2133

Santelices, R., Espinoza, S. y Cabrera, A. M. (2013). Efecto de distintos niveles de sombra y dosis de fertilizante en el cultivo en vivero de plantas de Nothofagus leonii Espinosa procedentes de su distribución más septentrional. En Sociedad Española de Ciencias Forestales (Ed.), Sexto congreso forestal español, pp. 1-9. Sociedad Española de Ciencias Forestales.

Skillman, J. B., García, M., Virgo, A. \& Winter, K. (2005). Growth irradiance effects on photosynthesis and growth in two co-occurring shade-tolerant neotropical perennials of contrasting photosynthetic pathways. American Journal of Botany, 92(11), 1811-1819. https://doi.org/10.3732/ ajb.92.11.1811

Taiz, L. \& Zeiger, E. (2010). Plant physiology. 5th ed. Sinauer Associates, Sunderland, MA 
Utumi, M., Monnerat, P., Gomes, P., Rezende, P. y Campos, V. (1999). Deficiência de macronutrientes em estévia: Sintomas visuais e efeitos no crescimento, composição química e produção de esteviosídeo. Pesquisa Agropecuária Brasileira, 34(6), 1039-1043. https://doi.org/10.1590/S0100204X1999000600016
Val, J., Heras, L., y Monge, E. (1985). Nuevas ecuaciones para la determinación de pigmentos fotosintéticos en acetona. Anales de la Estación Experimental de Aula Dei, 17(3-4), 231-238. http://hdl.handle. net/10261/13836 